\title{
Caracterização química de compostos e vermicompostos produzidos com casca de arroz e dejetos animais ${ }^{1}$
}

\author{
Elaine Luiza Biacchi Vione ${ }^{2 *}$, Leandro Souza da Silva ${ }^{3}$, Alberto Cargnelutti Filho, ${ }^{4}$ Natália Tobin Aita , \\ Alexssandro de Freitas de Morais ${ }^{5}$, Allan Augusto Kokkonen da Silva ${ }^{5}$
}

$10.1590 / 0034-737 X 201865010009$

\section{RESUMO}

Dejetos animais representam importantes fontes de nutrientes para as plantas; entretanto, necessitam de estabilização para evitar contaminação ambiental. Juntamente com a casca de arroz, podem ser usados em compostagens e vermicompostagens e reinseridos no sistema produtivo. Neste estudo, foram elaborados compostos e vermicompostos com casca de arroz e dejetos de aves, bovinos e suínos, que, após estabilização biológica, foram avaliados quanto à composição química e fracionamento de fibras, relacionando-se com sua adequação ao uso agrícola e seu potencial fertilizante. A redução do teor de $\mathrm{C}$ e da relação $\mathrm{C} / \mathrm{N}$ e o aumento da relação $\mathrm{CTC} / \mathrm{C}$ dos compostos e vermicompostos indicaram que os processamentos favoreceram a degradação dos resíduos orgânicos e sua estabilização. Os dejetos afetam a composição, sendo que maiores teores de $\mathrm{P}, \mathrm{Ca}$ e $\mathrm{Mg}$ foram obtidos nos materiais com dejetos de aves (1\%, $4,9 \%$ e $1,6 \%$, respectivamente). Os compostos e vermicompostos apresentaram composições similares, exceto para CE (redução de 1,6 para 0,5 dS.m ${ }^{-1}$ em vermicompostagem, na média dos três dejetos) e nos teores de K (redução de 1,8 para $0,6 \%$, em vermicompostagem com dejeto de aves), provavelmente pela lixiviação durante o processo. Houve maior fração solúvel $(61,5 \%)$ e menores teores de celulose $(9,3 \%)$ e lignina $(7,2 \%)$ no composto e vermicomposto com dejeto bovino, o que poderá favorecer a liberação de $\mathrm{N}$ para as plantas na decomposição desse material. Os dejetos de aves, bovinos e suínos resultaram em compostos maturados e vermicompostados com características químicas diferentes, sendo que os maiores teores de $\mathrm{P}, \mathrm{Ca}$ e $\mathrm{Mg}$ foram observados nos materiais produzidos com dejetos de aves. Concluise que os compostos e vermicompostos produzidos poderiam ser utilizados em substituição aos fertilizantes minerais e as quantidades a serem aplicadas devem ser ajustadas em função de sua composição específica.

Palavras-chave: análise química; fracionamento de fibras; reciclagem; sustentabilidade.

\section{ABSTRACT}

\section{Chemical characterization of compounds and vermicompost produced with rice hulls and animal manure}

Animal manure represents an important source of plant nutrients; however, it needs to be stabilized to avoid environmental contamination. These wastes, together with rice hulls, can be used in composting and vermicomposting and reinserted into the production system. In this study, compounds and vermicomposts were elaborated with rice hulls and poultry, bovine, and swine manure, which, after biological stabilization, were evaluated for chemical composition and fiber fractionation, associated to their suitability for agricultural use and potential fertilizer. The reduction in $\mathrm{C}$ content and $\mathrm{C} / \mathrm{N}$ ratio with increase in the CTC/C ratio of the compounds and vermicomposts indicated that the

\footnotetext{
Submetido em: 17/11/2016 e aprovado em: 01/02/2018.

' Parte da tese de doutorado da primeira autora. Programa de Pós-graduação em Ciência do Solo, Universidade Federal de Santa Maria, Santa Maria, Rio Grande do Sul. Brasil. ${ }^{2}$ Instituto Federal de Educação, Ciência e Tecnologia Farroupilha-Campus Santo Augusto, Santo Augusto, Rio Grande do Sul, Brasil. elaine.vione@iffaroupilha.edu.br

${ }^{3}$ Universidade Federal de Santa Maria, Centro de Ciências Rurais, Departamento de Solos, Santa Maria, Rio Grande do Sul, Brasil. leandrosolos@ufsm.br

${ }^{4}$ Universidade Federal de Santa Maria, Centro de Ciências Rurais, Departamento de Fitotecnia, Santa Maria, Rio Grande do Sul, Brasil. alberto.cargnelutti.filho@gmail.com

${ }^{5}$ Universidade Federal de Santa Maria, Santa Maria, Rio Grande do Sul, Brasil. natisa.aita@hotmail.com; alexssandro_freitas@hotmail.com; allan_kokkonen@hotmail.com

*Autor para correspondência: elainevione@yahoo.com.br
} 
processes boost the degradation of the organic residues and their stabilization. The manures affected the composition, with higher levels of $\mathrm{P}, \mathrm{Ca}$, and $\mathrm{Mg}$ in poultry waste materials (1, 4.9, and 1.6\%, respectively). The compounds and vermicomposts presented similar composition, except for EC (reduction of 1.6 to $0.5 \mathrm{dS} . \mathrm{m}^{-1}$ in vermicompost in the average of the three wastes) and in $\mathrm{K}$ contents (reduction 1.8 to $0.6 \%$ in vermicompost with poultry waste), probably due to leaching during the process. There was a higher soluble fraction (61.5\%) and lower cellulose (9.3\%) and lignin (7.2\%) contents in the compound and vermicompost with bovine manure, which could benefit the release of $\mathrm{N}$ to plants in the decomposition of this material. Compounds and vermicomposts could be used in sustainable production systems as a substitute for mineral fertilizers, and the amounts to be applied should be adjusted according to their specific composition.

Key words: chemical analysis; fiber fractionation; recycling; sustainability.

\section{INTRODUÇÃO}

A pecuária brasileira gera quantidade significativa de dejetos de animais (bovinos, suínos e aves), os quais, geralmente, têm sido diretamente aplicados aos solos como adubos orgânicos. Entretanto, aplicações frequentes de material in natura no mesmo local podem gerar problemas ambientais, como o excesso de P no solo (Scherer et al., 2010) e a lixiviação de nitrato (Aita \& Giacomini, 2008). Ao mesmo tempo, no processo de beneficiamento do arroz, outro resíduo significativo, a casca, gera um passivo ambiental.

Em sistemas agroecológicos e orgânicos, os fertilizantes minerais são excluídos e existe maior demanda por compostos orgânicos (Chalk et al., 2013). A compostagem e a vermicompostagem são os processos mais conhecidos e utilizados para a estabilização biológica de resíduos orgânicos e podem atender essa demanda, desde que respeitando a legislação. Adicionalmente, resultados de pesquisas indicam que a utilização do composto também diminui o estresse das plantas, pragas e doenças do solo, reduz alterações da temperatura do solo e a erosão, suprime plantas indesejáveis e aumenta a qualidade do solo (Moral et al., 2009). Além disso, o vermicomposto contém compostos semelhantes a hormônios ou reguladores que aceleram o crescimento e a produtividade das culturas (Domínguez \& Edwards, 2011).

A quantidade de um material orgânico a ser aplicada como fertilizante depende da sua composição, das culturas e das condições ambientais, sendo os critérios estabelecidos em boletins ou manuais de adubação (CQFS, 2016). Porém, o composto e o vermicomposto são, em geral, considerados como um produto específico e constante, sem levar em conta sua origem e, consequentemente, a composição química do material original. Entre os critérios de qualidade do composto são considerados o conteúdo de nutrientes, o grau de humificação, a percentagem de matéria orgânica estabilizada, o grau de maturidade e de higienização e a presença de compostos tóxicos, como metais pesados, sais solúveis e xenobióticos (Bernal et al., 2009). Esses parâmetros são afetados pela composição das matérias-primas utilizadas na sua produção, além das condições durante os processos em si.

Por isso, são necessários estudos sobre a sua composição, como subsídio para avaliar sua equivalência em fertilizante e os seus efeitos na composição da matéria orgânica e na produção de alimentos. O objetivo deste estudo foi avaliar a composição química de compostos e vermicompostos produzidos com casca de arroz e dejetos animais, visando a sua adequação ao uso agrícola e a determinar seu potencial fertilizante.

\section{MATERIAL E MÉTODOS}

O trabalho foi realizado na área experimental do Departamento de Solos da Universidade Federal de Santa Maria, RS (UFSM, RS), de agosto de 2014 a outubro de 2015. O experimento foi constituído da avaliação de três compostos e três vermicompostos, utilizando-se dejetos de bovinos, de suínos e de aves, misturados com casca de arroz. Anteriormente ao processo de compostagem, as matériasprimas foram homogeneizadas e amostradas para as análises químicas (Tabela 1).

A compostagem foi realizada em células de alvenaria com $0,95 \mathrm{~m}^{3}(1,04 \mathrm{~m} \times 1,30 \mathrm{~m} \times 0,7 \mathrm{~m}$ de altura), em que foram montadas pilhas de compostagem, com $70 \%$ (v/v) de casca de arroz como agente de volume e $30 \%$ (v/v) de dejeto (aves, bovinos ou suínos), intercaladas em camadas, sendo a primeira e a última camadas compostas pela casca de arroz. A mistura constou, em cada célula, de $350 \mathrm{~kg}$ de dejeto bovino e $84 \mathrm{~kg}$ de casca de arroz; de $285 \mathrm{~kg}$ de dejeto de suínos e $88 \mathrm{~kg}$ de casca de arroz; e de $306 \mathrm{~kg}$ de dejetos de aves e $88 \mathrm{~kg}$ de casca de arroz. A compostagem foi acompanhada por medições de temperatura e de umidade, por meio de um termo higrômetro e pelo "teste da umidade de mão" (Kiehl, 1985), mantendo-se a umidade em torno de $55 \%$. Realizaram-se eventos de aeração forçada a partir do quinto até o $23^{\circ}$ dia, por pelo menos $15 \mathrm{~min}$ 
dia $^{-1}$. Após esse período, aerações esporádicas foram realizadas, acompanhadas de revolvimento semanal, evitando-se o aumento excessivo da temperatura.

Após a etapa termofílica do processo de compostagem e estabilização da temperatura (60 dias), retirou-se uma alíquota do composto (aos 60 dias: C-60d), que foi congelada, e, após, metade do material foi destinado ao processo da vermicompostagem (V) e a outra metade do composto permaneceu em maturação $\left(\mathrm{C}_{\mathrm{m}}\right)$ nas células. $\mathrm{Na}$ vermicompostagem, foram utilizados canteiros de alvenaria de $0,33 \mathrm{~m}^{3}(0,95 \mathrm{~m} \times 0,98 \mathrm{~m} \times 0,35 \mathrm{~m}$ de altura), cobertos com telhas de zinco entremeadas com isopor e preenchidas, considerando-se um volume útil de $0,23 \mathrm{~m}^{3}$. Em cada canteiro, foi realizada a inoculação com minhocas da espécie Eisenia sp., inicialmente com 400 minhocas por canteiro (1.718 minhocas $\mathrm{m}^{-3}$ ) e, após 60 e 120 dias, mais 400 e 700 minhocas, cliteladas ou não, por canteiro, respectivamente. A vermicompostagem foi conduzida adicionando-se somente água para manutenção da umidade (80-90\%), utilizando-se em torno de 200 litros de água a mais do que no processo de compostagem + maturação. Aos 150 dias de vermicompostagem, quando se verificou que não houve mais alterações visuais no tamanho dos resíduos e as minhocas começaram a diminuir em número e tamanho rapidamente, encerrou-se o processo, retirando-se as minhocas manualmente e, concomitantemente, finalizou-se também a maturação do composto (total de 210 dias).

Ao final dos processos, composto maturado e vermicomposto prontos foram homogeneizados e embalados. Posteriormente, foi realizada a determinação do teor de umidade dos materiais pela secagem em estufa a $65^{\circ} \mathrm{C}$, até massa constante; a determinação do $\mathrm{pH}$ em água e em $\mathrm{CaCl}_{2}$ 0,01 mol L-1 (Tedesco et al., 1995); os teores de P, K, $\mathrm{Ca}$ e $\mathrm{Mg}$ foram determinados após digestão com $\mathrm{H}_{2} \mathrm{SO}_{4} \mathrm{e}$ $\mathrm{H}_{2} \mathrm{O}_{2}$ (Tedesco et al., 1995), sem uso da mistura de digestão, pois o $\mathrm{N}_{\text {total }}$ foi determinado em outro procedimento. Os teores de $\mathrm{Ca}$ e $\mathrm{Mg}$ foram determinados por espectrofotômetro de absorção atômica; o de P, por colorimetria (Murphy \& Riley, 1962), e, o de K, por fotometria de chama. As determinações foram realizadas em triplicata. Os teores de $\mathrm{N}$ e de $\mathrm{C}$ total foram determinados em analisador elementar (modelo Flash EA 1112, Termo Finnigan), em amostras previamente liofilizadas, moídas e maceradas a pó fino. Para avaliação da abundância isotópica do $\mathrm{N}\left(\delta^{15} \mathrm{~N}\right)$, as amostras foram sequencialmente analisadas por IRMS (Delta V Advantage, IRMS, Flash 2000 - EA for IRMS Thermo Scientific), e as determinações foram realizadas em duplicata. Também foram determinadas a CTC, em amostras em duplicata, e a condutividade elétrica (CE), em amostras em triplicata, utilizando-se a metodologia de Silva (2009), modificando-se na CE a centrifugação para dez minutos a $2000 \mathrm{rpm}$.
Os teores de $\mathrm{N}-\mathrm{NH}_{4}{ }^{+} \mathrm{e}$ de $\mathrm{N}-\mathrm{NO}_{3}{ }^{-}+\mathrm{NO}_{2}{ }_{2}^{-}$foram determinados após extração por destilação, em destilador semimicro Kjeldahl com arraste de vapor e determinação por titulação acidimétrica, com amostras em triplicata, exceto para os dejetos, para os quais foram determinados pelo skalar $S A N++$, com amostras em duplicata, tendo em vista a formação de precipitados e a dificuldade de mudança de cor na titulação com $\mathrm{H}_{2} \mathrm{SO}_{4}$. Nesses materiais, as amostras foram filtradas em papel filtro quantitativo de 2 $\mu \mathrm{m}$. Realizou-se também o fracionamento de fibras, com amostras em duplicata, determinando-se as frações solúvel, hemicelulose, celulose e lignina pelo método modificado de Van Soest (1963), com modificações nos procedimentos da determinação da fibra detergente neutro (FDN) e na determinação da lignina pelo ácido sulfúrico, alterando-se o período da queima em mufla por um período de dez horas, a $550^{\circ} \mathrm{C}$, 'por causa do elevado teor de lignina e sílica.

Os resultados analíticos foram submetidos a análise de variância $(\mathrm{P}<5 \%)$ e, quando significativo, as médias dos tratamentos foram comparadas pelo teste de Tukey, a $5 \%$ de probabilidade de erro, com o software estatístico SISVAR (Ferreira, 2014).

\section{RESULTADOS E DISCUSSÃO}

$\mathrm{O} \mathrm{pH}_{\mathrm{H} 2 \mathrm{O}}$ variou de 6,5 a 8,2, nos compostos $\left(\mathrm{C}_{\mathrm{m}}\right)$, e de 6,9 a 8,1 nos vermicompostos $(\mathrm{V}) . \mathrm{O} \mathrm{pH}_{\mathrm{CaCl} 2}$ variou de $6,2 \mathrm{a}$ 7,6 , nos $\mathrm{C}_{\mathrm{m}}$ e de 6,4 a 7,3, nos V, o que está de acordo com Brasil (2009), que estabelece um pH mínimo de 6,0. O pH ideal do composto deve ser de ligeiramente ácido a neutro; valores superiores a 8,4 podem ser prejudiciais às plantas, principalmente se associados a odores e a perda de $\mathrm{NH}_{3}$ (Woods End, 2005). Os maiores valores de $\mathrm{pH}$ foram verificados na utilização de dejetos de aves, podendo resultar em maiores emissões de $\mathrm{NH}_{3}$ (Sun et al., 2014), por causa da concentração inicial de $\mathrm{NH}_{4}^{+}$(Tabela 1) e da presença de compostos facilmente mineralizáveis, como o ácido úrico e o chorume, no esterco de galinha (Bernal et al., 1998). Além disso, o agente de volume adicionado ao dejeto pode influenciar nas perdas de N (Sun et al., 2014), uma vez que a casca de arroz, com pouco N (Tabela 1) e mais rica em celulose e lignina, promove a imobilização do $\mathrm{N}$ e, consequentemente, reduz as emissões de $\mathrm{NH}_{3}$ (Zeng et al., 2012).

Geralmente, os dejetos apresentam um valor médio de $\delta^{15} \mathrm{~N}$ de $7,7 \%$ (Tabela 1), e uma amplitude de 2,9\%o a 16,2\%。 (Bateman \& Kelly, 2006). Porém, esses valores sofreram alterações quando as matérias-primas foram misturadas com a casca, apresentando, no C-60d, $\mathrm{C}_{\mathrm{m}}$ e no $\mathrm{V}$, valores médios de $\delta^{15} \mathrm{~N}$ de $11,1,11,3$ e $10,5 \%$ o, respectivamente. Após o processamento, diferenciaram-se com o tipo de dejeto utilizado, com valores de $\delta^{15} \mathrm{~N} 13,5 \%$, para os deri- 
vados de dejeto de aves, 8,4\%o, para os de dejetos bovinos e 11,1\%o, para os de dejetos suínos, salientando-se o maior $\delta^{15} \mathrm{~N}$ na utilização de dejeto de aves. As diferenças podem ser devidas a uma combinação de mecanismos de fracionamento isotópico, incluindo a discriminação isotópica microbiana durante a ciclagem do $\mathrm{N}$, uma mudança para compostos nitrogenados mais complexos e ao fracionamento isotópico durante a volatilização de $\mathrm{NH}_{3}$ (Lynch et al., 2006), além de diferenças na volatilização de $\mathrm{NH}_{3}$ entre os compostos, que poderiam ser afetados por

Tabela 1: Caracterização química das matérias-primas utilizadas na elaboração dos compostos e vermicompostos

\begin{tabular}{|c|c|c|c|c|c|}
\hline Parâmetro & Casca de arroz & Dejetos de aves & Dejetos de suínos & Dejetos de bovinos & $\mathrm{CV}(\%)$ \\
\hline $\mathrm{C}(\%)$ & $35,2 \mathrm{a}^{*}$ & $26,0 \mathrm{~b}$ & $26,6 \mathrm{~b}$ & $17,4 \mathrm{c}$ & 7,42 \\
\hline $\mathrm{N}_{\text {total }}(\%)$ & $1,62 \mathrm{~b} \mathrm{c}$ & $3,38 \mathrm{a}$ & $2,30 \mathrm{~b}$ & $1,24 \mathrm{c}$ & 10,61 \\
\hline $\mathrm{C} / \mathrm{N}$ & $22 \mathrm{a}$ & $8,0 \mathrm{c}$ & $12 \mathrm{bc}$ & $14 \mathrm{~b}$ & 9,97 \\
\hline $\mathrm{N}-\mathrm{NH}_{4}^{+}(\%)$ & $<0,01 \mathrm{~d}$ & $0,52 \mathrm{a}$ & $0,16 b$ & $0,06 \mathrm{c}$ & 4,93 \\
\hline $\mathrm{N}-\mathrm{NO}_{3}^{-}+\mathrm{NO}_{2}^{-}(\%)$ & $<0,01$ & $<0,01$ & $<0,01$ & $<0,01$ & - \\
\hline$\delta{ }^{15} \mathrm{~N}(\%)$ & $11,33 \mathrm{a}$ & $5,71 \mathrm{~b}$ & $6,80 \mathrm{~b}$ & $7,32 \mathrm{ab}$ & 13,45 \\
\hline $\mathrm{P}(\%)$ & $0,05 \mathrm{c}$ & $1,27 \mathrm{a}$ & $0,68 \mathrm{~b}$ & $0,65 \mathrm{~b}$ & 4,12 \\
\hline $\mathrm{K}(\%)$ & $0,26 \mathrm{c}$ & $2,46 \mathrm{a}$ & $1,06 \mathrm{~b}$ & $1,21 \mathrm{~b}$ & 5,4 \\
\hline $\mathrm{Ca}(\%)$ & $0,13 \mathrm{~b}$ & $6,11 \mathrm{a}$ & $0,52 \mathrm{~b}$ & $1,09 \mathrm{~b}$ & 69,88 \\
\hline $\operatorname{Mg}(\%)$ & $0,37 \mathrm{~d}$ & $2,13 \mathrm{a}$ & $1,68 \mathrm{~b}$ & $1,48 \mathrm{c}$ & 5,11 \\
\hline $\mathrm{Na}(\%)$ & $0,02 \mathrm{c}$ & $0,33 \mathrm{a}$ & $0,07 \mathrm{~b} \mathrm{c}$ & $0,11 \mathrm{~b}$ & 16,8 \\
\hline Fração solúvel (\%) & $20,8 *$ & - & - & - & - \\
\hline Celulose (\%) & $24,7^{*}$ & - & - & - & - \\
\hline Hemicelulose (\%) & $44,6^{*}$ & - & - & - & - \\
\hline Lignina & $9,8^{*}$ & - & - & - & - \\
\hline Celulose/Lignina & 2,5 & - & - & - & - \\
\hline
\end{tabular}

${ }^{*}$ Médias seguidas de mesma letra minúscula na linha não diferem a 5\% de significância pelo teste de Tukey. *Valores correspondentes a MS a $105^{\circ} \mathrm{C}$.

Tabela 2: Caracterização química do composto, aos 60 dias (C-60d) e maturado ( $\mathrm{C}_{\mathrm{m}}=210$ dias), e do vermicomposto ( $\mathrm{V}=210$ dias), produzidos a partir de casca de arroz e de diferentes tipos de dejetos animais

\begin{tabular}{|c|c|c|c|c|}
\hline Dejeto & C-60d & $\mathrm{C}_{\mathrm{m}}$ & $\mathbf{V}$ & Média geral \\
\hline & \multicolumn{4}{|c|}{$\mathrm{N}$ total $(\%)$} \\
\hline Aves & 1,00 & 1,3 & 1,3 & $1,2 \mathrm{~b}$ \\
\hline Bovinos & 1,00 & 1,2 & 1,1 & $1,1 \mathrm{~b}$ \\
\hline Suínos & 1,22 & 1,5 & 1,5 & $1,4 \mathrm{a}$ \\
\hline Média geral & $1,07 \mathrm{~B}^{*}$ & $1,3 \mathrm{~A}$ & $1,3 \mathrm{~A}$ & \\
\hline \multirow[t]{2}{*}{$\mathrm{CV}(\%)$} & 5,48 & & & \\
\hline & \multicolumn{4}{|c|}{$\mathrm{N}-\mathrm{NO}_{3}{ }^{-}+\mathrm{N}-\mathrm{NO}_{2}^{-}(\%)$} \\
\hline Aves & $0,02 \mathrm{~A} \mathrm{~B} \mathrm{a}$ & $0,02 \mathrm{Ab}$ & $0,01 \mathrm{~B} \mathrm{a}$ & \\
\hline Bovinos & $<0,01 \mathrm{C} \mathrm{b}$ & $0,04 \mathrm{~A} \mathrm{a}$ & $0,02 \mathrm{~B} \mathrm{a}$ & \\
\hline Suínos & $0,01 \mathrm{~B} \mathrm{~b}$ & $0,03 \mathrm{Ab}$ & $0,01 \mathrm{~B} \mathrm{a}$ & \\
\hline \multirow[t]{2}{*}{$\mathrm{CV}(\%)$} & 35,15 & & & \\
\hline & \multicolumn{4}{|c|}{$\mathbf{P}(\%)$} \\
\hline Aves & 0,9 & 1,1 & 1,00 & $1,00 \mathrm{a}$ \\
\hline Bovinos & 0,3 & 0,5 & 0,4 & $0,4 \mathrm{~b}$ \\
\hline Suínos & 0,5 & 0,5 & 0,4 & $0,5 \mathrm{~b}$ \\
\hline \multirow[t]{2}{*}{$\mathrm{CV}(\%)$} & 12,77 & & & \\
\hline & \multicolumn{4}{|c|}{$\mathrm{Ca}(\%)$} \\
\hline Aves & $2,2 \mathrm{~B} \mathrm{a}$ & $4,8 \mathrm{~A} \mathrm{a}$ & $4,9 \mathrm{~A} \mathrm{a}$ & \\
\hline Bovinos & $0,7 \mathrm{Ab}$ & $1,2 \mathrm{Ab}$ & $1,1 \mathrm{Ab}$ & \\
\hline Suínos & $0,5 \mathrm{Ab}$ & $0,5 \mathrm{Ab}$ & $0,6 \mathrm{Ab}$ & \\
\hline $\mathrm{CV}(\%)$ & 33,49 & & & \\
\hline
\end{tabular}


condições da compostagem e pelos materiais utilizados (Lim et al., 2010).

Os teores de $\mathrm{N}$ total (Tabela 2) no $\mathrm{C}_{\mathrm{m}}$ e no $\mathrm{V}$ foram maiores que $1,0 \%$, superiores ao mínimo de $0,5 \%$ estabelecido (Brasil, 2009). Contudo, os teores de $\mathrm{NH}_{4}{ }^{+}$nos diferentes $\mathrm{C}_{\mathrm{m}}$ ou $\mathrm{V}$ foram muito baixos $(<0,01 \%)$, enquanto maiores teores de $\mathrm{NO}_{3}{ }^{-}+\mathrm{NO}_{2}{ }^{-}$foram verificados nos $\mathrm{C}_{\mathrm{m}} \mathrm{de}$ aves $(0,02 \%)$, bovinos $(0,04 \%)$ e suínos $(0,03 \%)$, em comparação com os $\mathrm{V}$ das mesmas matérias-primas $(0,01,0,02 \mathrm{e}$ $0,01 \%$, respectivamente). Mas, independentemente de serem obtidos por compostagem ou vermicompostagem, os teores de $\mathrm{NO}_{3}{ }^{-}+\mathrm{NO}_{2}{ }^{-}$foram superiores àqueles do $\mathrm{C}-60 \mathrm{~d}$ (Tabela 2) Consequentemente, a relação $\mathrm{NH}_{4}^{+} / \mathrm{NO}_{3}^{-}$, embora superior no $\mathrm{C}_{\mathrm{m}}$ e no $\mathrm{V}$ suínos $(0,05$ e 0,18 , respectivamente), foi inferior a 1,0, indicando a estabilização dos materiais no final do período (Bernal et al., 1998).

Para o Ca, os teores aumentaram no $\mathrm{C}_{\mathrm{m}}(4,8 \%)$ e no V $(4,9 \%)$ de aves, em comparação com os do C-60d (2,2\%), enquanto não se alteraram com os dejetos de bovinos e suínos (Tabela 2). Resultados similares ao do Ca foram observados para o P (Tabela 2), com maiores teores quando utilizados dejetos de aves $(1,0 \%)$. Porém, o teor de $\mathrm{Mg}$ (Tabela 3) não diferiu entre $\mathrm{C}_{\mathrm{m}} \mathrm{e} \mathrm{V}(1,4 \%)$, mas foi superior ao do C-60d (1,2\%). Os maiores teores de $\mathrm{P}$ e de $\mathrm{Ca}$, com a utilização do dejeto de aves poedeiras (Tabela 1) devemse à adição dos elementos por meio dos minerais nas rações. Esses resultados estão de acordo com os de Swarnam et al. (2016), que, após a vermicompostagem a partir da casca de coco com esterco de aves, produziram um vermicomposto mais rico em $\mathrm{Ca}$ do que a partir do esterco de suínos. O teor de $\mathrm{K}$ aumentou durante a compostagem, quando utilizado dejeto de aves (1,3\%, no C-60d, para 1,8\%, no $\left.\mathrm{C}_{\mathrm{m}}\right)$, mas reduziu-se durante a vermicompostagem $(0,6 \%)$. O mesmo ocorreu com composto bovino, $(0,7 \%$, no C-60d, para $0,8 \%$, no $\mathrm{C}_{\mathrm{m}}$ ) e na $\mathrm{V}$, reduziu-se para $0,4 \%$. Porém, no de suínos não se alterou com a compostagem $(0,7 \%$, no C60d e no $\mathrm{C}_{\mathrm{m}}$ ), mas reduziu-se com a vermicompostagem $(0,4 \%)$ (Tabela 3$)$. A redução do K com a $\mathrm{V}$ pode ser atribuída à lixiviação do elemento por causa da maior adição de água no processo e sua presença na forma iônica $\left(\mathrm{K}^{+}\right)$ (Frederickson et al., 2007). Entre os diferentes dejetos, o de aves contribuiu para o maior teor de $\mathrm{K}_{\text {no }} \mathrm{C}_{\mathrm{m}}(1,8 \%)$ (Tabela 3). De modo similar, o teor de sódio (Na), no composto de aves (Tabela 3$)$, foi maior no $\mathrm{C}_{\mathrm{m}}(0,24 \%)$ do que no C-60d $(0,17 \%)$, porém, diminuiu no $\mathrm{V}(0,06 \%)$, mas encontra-se em níveis adequados, pois acima de $1 \%$ poderia ser tóxico para o sistema radicular, afetando a germinação e a emergência de várias espécies de plantas além de com-

Tabela 3: Caracterização química e valores de CE do composto, aos 60 dias (C-60d) e maturado ( $\mathrm{C}_{\mathrm{m}}=210$ dias), e do vermicomposto ( $\mathrm{V}=210$ dias), produzidos a partir de casca de arroz e de diferentes tipos de dejetos animais

\begin{tabular}{|c|c|c|c|c|}
\hline Dejeto & C-60d & $\mathrm{C}_{\mathrm{m}}$ & $\mathbf{V}$ & Média geral \\
\hline & \multicolumn{4}{|c|}{$\mathbf{K}(\%)$} \\
\hline Aves & $1,3 \mathrm{~B} \mathrm{a}^{*}$ & $1,8 \mathrm{Aa}$ & $0,6 \mathrm{C} \mathrm{a}$ & \\
\hline Bovinos & $0,7 \mathrm{Ab}$ & $0,8 \mathrm{Ab}$ & $0,4 \mathrm{~B} \mathrm{~b}$ & \\
\hline Suínos & $0,7 \mathrm{Ab}$ & $0,7 \mathrm{Ab}$ & $0,4 \mathrm{~B} \mathrm{~b}$ & \\
\hline \multirow[t]{2}{*}{ CV $(\%)$} & 8,56 & & & \\
\hline & \multicolumn{4}{|c|}{$\mathrm{Na}(\%)$} \\
\hline Aves & $0,17 \mathrm{~B} \mathrm{a}$ & $0,24 \mathrm{Aa}$ & $0,06 \mathrm{C} \mathrm{a}$ & \\
\hline Bovinos & $0,06 \mathrm{Ab}$ & $0,06 \mathrm{~A} \mathrm{~b}$ & $0,04 \mathrm{~A} \mathrm{a}$ & \\
\hline Suínos & $0,06 \mathrm{Ab}$ & $0,04 \mathrm{~A} \mathrm{~b}$ & $0,06 \mathrm{~A} \mathrm{a}$ & \\
\hline \multirow[t]{2}{*}{$\mathrm{CV}(\%)$} & 21,79 & & & \\
\hline & \multicolumn{4}{|c|}{$\operatorname{Mg}(\%)$} \\
\hline Aves & 1,4 & 1,7 & 1,7 & $1,6 \mathrm{a}$ \\
\hline Bovinos & 0,9 & 1,2 & 1,2 & $1,1 \mathrm{~b}$ \\
\hline Suínos & 1,2 & 1,2 & 1,2 & $1,2 \mathrm{~b}$ \\
\hline Média geral & $1,2 \mathrm{~B}$ & $1,4 \mathrm{~A}$ & $1,4 \mathrm{~A}$ & \\
\hline \multirow[t]{2}{*}{$\mathrm{CV}(\%)$} & 9,60 & & & \\
\hline & \multicolumn{4}{|c|}{ CE $\left(d S . m^{-1}\right)$} \\
\hline Aves & nd & 1,8 & 0,60 & $1,2 \mathrm{a}$ \\
\hline Bovinos & nd & 1,6 & 0,50 & $1,1 \mathrm{a}$ \\
\hline Suínos & nd & 1,3 & 0,4 & $0,9 \mathrm{~b}$ \\
\hline Média geral & nd & $1,6 \mathrm{~A}$ & $0,5 \mathrm{~B}$ & \\
\hline $\mathrm{CV}(\%)$ & 10,89 & & & \\
\hline
\end{tabular}


petir, na absorção, com Ca e K (A\&L Canada Laboratories, 2004). Sua redução com a vermicompostagem também está associada com a lixiviação de $\mathrm{Na}$, à semelhança do que ocorreu com o $\mathrm{K}$.

A maior condutividade elétrica (CE) do $\mathrm{C}_{\mathrm{m}}\left(1,6 \mathrm{dS} \mathrm{\textrm {m } ^ { - 1 } )}\right.$ (Tabela 3$)$, em comparação com a do V $\left(0,5 \mathrm{dS} . \mathrm{m}^{-1}\right)$, indica níveis mais elevados de nutrientes no composto (Frederickson et al., 2007), que podem ser devidos à liberação de sais de amônio solúveis e de fosfatos, com a degradação de compostos mais lábeis na fase termofílica da compostagem, e à maior lixiviação, na vermicompostagem (Lazcano et al., 2008). Além disso, a CE reflete a salinidade dos resíduos orgânicos que, em alta concentração, podem causar fitotoxicidade, sendo um parâmetro da adequação e segurança da utilização dos compostos/vermicompostos para fins agrícolas (Lazcano et al., 2008), de modo que compostos com valores de CE entre $0,75-2,0 \mathrm{dS} \cdot \mathrm{m}^{-1}$ podem ser utilizados diretamente como meio de cultivo para plântulas e em sementeiras (A\&L Canada Laboratories, 2004).

$\mathrm{O}$ teor de carbono (C) (Tabela 4) foi maior no C-60d $(25,6 \%)$, reduzindo-se com a $\mathrm{C}_{\mathrm{m}}(19,8 \%)$ e a V $(20,5 \%)$. Maiores teores de $\mathrm{C}$ foram verificados nos materiais que utilizaram dejetos de suínos (24,6\%), em comparação com os dos dejetos de aves $(23,2 \%)$ ou bovinos $(18,2 \%)$. Durante a decomposição ocorrem perdas de $\mathrm{C}$, principalmente na forma de $\mathrm{CO}_{2}$, e o revolvimento favorece essas perdas, por maior oxigenação. Possivelmente, uma redução na frequência do revolvimento amenizaria as perdas de $\mathrm{C}$ (Santos et al., 2010), mas o teor de C obtido está em consonância com o mínimo 15\%, para composto, e de $10 \%$, para vermicomposto (Brasil, 2009).

Valores mais elevados de $\mathrm{C}$ orgânico estão relacionados com valores mais baixos de CTC, refletindo menor grau de humificação (Rodella \& Alcarde, 1994). Porém, verificou-se um aumento da CTC (Tabela 4) com a compostagem e com a vermicompostagem, sendo este um parâmetro indicativo da qualidade e da capacidade em reter nutrientes quando incorporados ao solo (Rodella \& Alcarde, 1994). A relação CTC/C foi maior quando utilizado dejeto bovino $(19,4)$ e menor quando utilizado dejeto suíno $(14,5)$, porém, não foi influenciada pela forma de processamento (compostagem ou vermicompostagem).

Não houve diferença na relação C/N entre o $C_{m}(14,9)$ e o V $(15,8)$, mas foi maior no C-60d $(24,3)$ e quando se utili-

Tabela 4: Teores de C, CTC, relação C/N e CTC/C do composto, aos 60 dias (C-60d) e maturado $\left(\mathrm{C}_{\mathrm{m}}=210\right.$ dias), e do vermicomposto ( $\mathrm{V}=210$ dias), produzidos a partir de casca de arroz e de diferentes tipos de dejetos animais

\begin{tabular}{|c|c|c|c|c|}
\hline Dejeto & C-60d & $\mathbf{C}_{\mathrm{m}}$ & $\mathbf{V}$ & Média Geral \\
\hline & \multicolumn{4}{|c|}{$\mathrm{C}(\%)$} \\
\hline Aves & 27,1 & 22,2 & 20,2 & $23,2 \mathrm{~b}$ \\
\hline Bovinos & 21,8 & 15,8 & 17,0 & $18,2 \mathrm{~b}$ \\
\hline Suínos & 28,0 & 21,4 & 24,4 & $24,6 \mathrm{a}$ \\
\hline Média geral & $25,6 \mathrm{~A}^{*}$ & $19,8 \mathrm{~B}$ & $20,5 \mathrm{~B}$ & \\
\hline \multirow[t]{2}{*}{$\mathrm{CV}(\%)$} & 4,45 & & & \\
\hline & \multicolumn{4}{|c|}{ CTC (mmol kg-1) } \\
\hline Aves & 190,0 & 381,3 & 358,8 & 310,0 \\
\hline Bovinos & 223,8 & 333,8 & 301,3 & 286,3 \\
\hline Suínos & 200,0 & 328,8 & 332,5 & 287,1 \\
\hline Média geral & 204,6 B & $347,9 \mathrm{~A}$ & $330,8 \mathrm{~A}$ & \\
\hline \multirow[t]{2}{*}{$\mathrm{CV}(\%)$} & 13,25 & & & \\
\hline & \multicolumn{4}{|c|}{ Relação C/N } \\
\hline Aves & 28 & 16,6 & 16,1 & $20,3 \mathrm{a}$ \\
\hline Bovinos & 21,8 & 13,4 & 15,4 & $16,9 \mathrm{~b}$ \\
\hline Suínos & 23,2 & 14,5 & 15,9 & $17,9 \mathrm{~b}$ \\
\hline Média geral & $24,3 \mathrm{~A}$ & $14,9 \mathrm{~B}$ & $15,8 \mathrm{~B}$ & \\
\hline \multirow[t]{2}{*}{$\mathrm{CV}(\%)$} & 7,31 & & & \\
\hline & \multicolumn{4}{|c|}{ Relação CTC/C } \\
\hline Aves & 7,0 & 17,1 & 17,6 & $13,9 \mathrm{ab}$ \\
\hline Bovinos & 10,4 & 21,1 & 17,7 & $16,4 \mathrm{a}$ \\
\hline Suínos & 8,3 & 15,4 & 13,7 & $12,4 \mathrm{~b}$ \\
\hline Média Geral & $8,6 \mathrm{~B}$ & $17,9 \mathrm{~A}$ & $16,3 \mathrm{~A}$ & \\
\hline $\mathrm{CV}(\%)$ & 11,44 & & & \\
\hline
\end{tabular}


zou dejeto de aves $(20,3)$ (Tabela 4$)$. A relação C/N é um parâmetro variável, dependente das matérias-primas, sendo ideal que seja próxima a 10, mas, por causa de compostos orgânicos recalcitrantes ou de lenta decomposição, são aceitos valores de relação $\mathrm{C} / \mathrm{N}$ maiores em compostos maduros e em vermicompostos (Domínguez \& Edwards, 2011). A relação $\mathrm{C} / \mathrm{N}$ média para $\mathrm{o}_{\mathrm{m}}$ foi de $14,9 \mathrm{e}$, para o $\mathrm{V}$, de 15,8; porém, segundo Brasil (2009), a relação C/N máxima para composto é 20 e para o vermicomposto é 14 e, dessa forma, $\mathrm{o}_{\mathrm{m}}$ estaria nos padrões estabelecidos, porém, o $\mathrm{V}$ ainda não. A maior relação $\mathrm{C} / \mathrm{N}$ no $\mathrm{V}$ poderia ser atribuída a menor degradação realizada pelas minhocas em materiais com maior quantidade de lignina e sílica, como a casca de arroz (Cotta et al., 2015). Entretanto, resíduos orgânicos com relação $\mathrm{C} / \mathrm{N}$ similares podem mineralizar diferentes quantidades de $\mathrm{N}$, por causa de diferenças na composição, não refletidas por essa relação (Cabrera et al., 2005). Possivelmente, a relação CTC/C seria mais adequada que a relação $\mathrm{C} / \mathrm{N}$ para indicar o grau de humificação (Dores-Silva et al., 2013).

No fracionamento de fibras, maiores frações solúveis (Tabela 5) foram verificadas no $\mathrm{C}_{\mathrm{m}}(63 \%)$ e no $\mathrm{V}(60,6 \%)$, em comparação com as do C-60d (46,2\%). Além disso, elas foram maiores quando utilizados dejetos de bovinos $(61,5 \%)$. A similaridade da fração solúvel entre o $\mathrm{C}_{\mathrm{m}} \mathrm{e} \mathrm{V}$ (Tabela 5) indica que os processos foram eficientes e equivalentes, aumentando a solubilização dos materiais, o que se torna ainda mais evidente ao se considerar a casca de arroz (Tabela 1). Entretanto, Ngo et al. (2013) encontraram maior solubilidade em vermicompostos do que em compostos de mesma matéria-prima.

Os teores de celulose (Tabela 5) no composto produzido com dejeto de aves foram maiores no C-60d (17,9\%), diminuindo após a $\mathrm{C}_{\mathrm{m}}(10,8 \%)$, com os menores teores quando no $\mathrm{V}(8,4 \%)$. Entretanto, maiores teores foram verificados no $\mathrm{C}_{\mathrm{m}}$ de aves $(10,8 \%)$ e os menores no $\mathrm{C}_{\mathrm{m}}$ bovino $(5,5 \%)$. O menor teor de celulose no $\mathrm{C}_{\mathrm{m}}$ bovino pode ser devido aos micro-organismos existentes no rúmen que produzem enzimas celulases e hemicelulases (Malik et al., 2015), enquanto, na vermicompostagem, a utilização do C pelos micro-organismos, a ação das minhocas e a interação desses organismos podem ter favorecido a degradação da celulose (Gómez-Brandón et al., 2010).

O teor de hemicelulose foi semelhante para $\mathrm{C}_{\mathrm{m}}(20,7 \%)$ e V $(23,1 \%)$, embora menores que no C-60 d (29,3\%) (Tabela 5). A degradação da hemicelulose pode ser atribuída aos

Tabela 5: Fracionamento de fibras do composto, aos 60 dias (C-60d) e maturado $\left(\mathrm{C}_{\mathrm{m}}=210\right.$ dias), e do vermicomposto (V=210 dias), produzidos a partir de casca de arroz e de diferentes tipos de dejetos animais

\begin{tabular}{|c|c|c|c|c|}
\hline Dejeto & C-60d & $\mathrm{C}_{\mathrm{m}}$ & $\mathbf{V}$ & Média geral \\
\hline \multicolumn{5}{|c|}{ Fração solúvel (\%) } \\
\hline Aves & 45,2 & 58,2 & 59,1 & $54,2 \mathrm{~b}$ \\
\hline Bovinos & 47,3 & 72,4 & 64,8 & $61,5 \mathrm{a}$ \\
\hline Suínos & 46,0 & 58,4 & 57,8 & $54,1 \mathrm{~b}$ \\
\hline Média geral & $46,2 \mathrm{~B}^{*}$ & $63,0 \mathrm{~A}$ & $60,6 \mathrm{~A}$ & \\
\hline \multirow[t]{2}{*}{$\mathrm{CV}(\%)$} & 4,93 & & & \\
\hline & \multicolumn{4}{|c|}{ Celulose (\%) } \\
\hline Aves & $17,9 \mathrm{~A} \mathrm{a}$ & $10,8 \mathrm{~B} \mathrm{a}$ & $8,4 \mathrm{C} \mathrm{a}$ & 12,3 \\
\hline Bovinos & $15,5 \mathrm{Ab}$ & $5,5 \mathrm{~B} \mathrm{c}$ & $7,1 \mathrm{~B} \mathrm{a}$ & 9,3 \\
\hline Suínos & $14,8 \mathrm{~A} \mathrm{~b}$ & $8,2 \mathrm{~B} \mathrm{~b}$ & $8,9 \mathrm{~B} \mathrm{a}$ & 10,6 \\
\hline Média geral & 16,1 & 8,2 & 8,1 & \\
\hline \multirow[t]{2}{*}{$\mathrm{CV}(\%)$} & 6,39 & & & \\
\hline & \multicolumn{4}{|c|}{ Hemicelulose (\%) } \\
\hline Aves & 28,5 & 22,4 & 24,8 & 25,2 \\
\hline Bovinos & 29,5 & 15,7 & 20,9 & 22,0 \\
\hline Suínos & 30,0 & 24 & 23,6 & 25,9 \\
\hline Média geral & $29,3 \mathrm{~A}$ & $20,7 \mathrm{~B}$ & $23,1 \mathrm{~B}$ & \\
\hline \multirow[t]{2}{*}{$\mathrm{CV}(\%)$} & 11,55 & & & \\
\hline & \multicolumn{4}{|c|}{ Lignina (\%) } \\
\hline Aves & 8,4 & 8,6 & 7,7 & $8,3 \mathrm{~b}$ \\
\hline Bovinos & 7,8 & 6,5 & 7,3 & $7,2 \mathrm{c}$ \\
\hline Suínos & 9,1 & 9,4 & 9,7 & $9,4 \mathrm{a}$ \\
\hline Média geral & 8,5 & 8,2 & 8,2 & \\
\hline $\mathrm{CV}(\%)$ & 5,74 & & & \\
\hline
\end{tabular}


micro-organismos que são capazes de produzir enzimas celulases e às xilases que degradam a celulose e a hemicelulose (Wu et al., 2015).

Maior teor de lignina (Tabela 5) foi verificado quando utilizado dejeto de suínos $(9,40 \%)$ e menor com dejeto de bovinos $(7,2 \%)$, tanto em compostagem quanto em vermicompostagem. $\mathrm{O}$ menor teor de lignina com dejeto bovino poderia ser devido à presença de fungos anaeróbicos no rúmen de bovinos que produzem enzimas capazes de degradar parcial ou totalmente tecidos lignificados (Gruninger et al., 2014). A degradação da lignina em condições aeróbicas poderia ser realizada também por fungos da podridão branca e parda e, em menor escala, por bactérias (Tuomela et al., 2000; Komilis \& Ham, 2003). Porém, na compostagem, a degradação da lignina seria influenciada pela temperatura, pelo teor inicial de lignina e pela espessura do material (Tuomela et al., 2000).

Paralelamente, a relação celulose/lignina foi maior quando utilizados dejetos de aves $(1,5)$, e menor, quando utilizados dejetos de suínos $(1,1)$. Entretanto, durante o processamento, foi maior no C-60d $(1,92)$ e menor em $\mathrm{C}_{\mathrm{m}} \mathrm{e}$ $\mathrm{V}(0,99)$, não se diferenciando entre os processos. A relação celulose/lignina pode ser um indicativo da evolução do processo de compostagem (Paradelo et al., 2013) e a menor relação celulose/lignina (Tabela 5) pode sugerir um material biologicamente mais estável, evidenciado pela maior fração residual do método de Van Soest, composta de lignina e outras moléculas aromáticas e alifáticas não solúveis (Ngo et al., 2013).

Assim, os potenciais de decomposição e de liberação de $\mathrm{N}$ de materiais orgânicos adicionados aos solos estão relacionados com sua composição bioquímica, em que a concentração de $\mathrm{N}$, a relação $\mathrm{C} / \mathrm{N}$, e o teor de lignina, parecem ser importantes na previsão da mineralização do N dos resíduos (Abbasi et al., 2015).

\section{CONCLUSÕES}

Os dejetos de aves, bovinos e suínos resultaram em compostos maturados e vermicompostados com características químicas diferentes. Maiores teores de $\mathrm{P}, \mathrm{Ca}$ e $\mathrm{Mg}$ foram observados nos materiais produzidos com dejetos de aves. Os produtos obtidos com a compostagem e a vermicompostagem, após 60 dias de pré-compostagem, foram similares, exceto para a CE e os teores de K, que foram menores no vermicomposto. A maior fração solúvel e os menores teores de celulose, lignina e relação celulose/lignina no composto maturado e no vermicomposto, quando utilizado dejeto bovino, poderá favorecer a liberação de $\mathrm{N}$ para as plantas na decomposição desse material. Os compostos e vermicompostos produzidos poderiam ser utilizados em sistemas de produção sustentáveis, em substituição aos fertilizantes minerais e as quantida- des a serem aplicadas devem ser ajustadas em função de sua composição específica.

\section{AGRADECIMENTOS}

Os autores agradecem aos professores Zaida I. Antoniolli, Rodrigo J.S. Jacques, Sandro J. Giacomini e Leila P. da Silva e aos alunos Gerson L. Drescher, Cesar Cella, Laila G. Marques e Luis H. F. Prigol, pelo apoio na condução e análises do experimento, e ao CNPq, pela concessão de bolsa de Produtividade em Pesquisa.

\section{REFERÊNCIAS}

Abbasi MK, Tahir MM, Sabir N \& Khurshid M (2015) Impact of the addition of different plant residues on nitrogen mineralizationimmobilization turnover and carbon content of a soil incubated under laboratory conditions. Solid Earth, 6:197-205.

Aita C \& Giacomini SJ (2008) Nitrato no solo com a aplicação de dejetos líquidos de suínos no milho em plantio direto. Revista Brasileira de Ciência do Solo, 32:2101-2111.

A\&L Canada Laboratories Inc (2004) Compost Management Program. Disponível em: <http://www.alcanada.com/ index_htm_files/Compost_Handbook.pdf>. Acessado em: 31 de março de 2016.

Bateman A \& Kelly S (2006) Discriminating between organically and conventionally grown crops using stable isotope and multielement analysis. Final report on 3-year project +6 -month extension. UEA - Norwich/Institute of Food Research - Colney, Norwich. 151p.

Bernal MP, Paredes C, Sánchez-Monedero MA \& Cegarra J (1998) Maturity and stability parameters of composts prepared with a, wide range of organic wastes. Bioresource Technology, 63:9199.

Bernal MP, Alburquerque, JA \& Moral R (2009) Composting of animal manures and chemical criteria for compost maturity assessment: A review. Bioresource Technology, 100:5444-5453.

Brasil (2009) Instrução Normativa $n^{\circ} 25$, de 23 de julho de 2009. Normas sobre as especificações e as garantias, as tolerâncias, o registro, a embalagem e a rotulagem dos fertilizantes orgânicos simples, mistos, compostos, organominerais e biofertilizantes destinados à agricultura. DOU, 28/07/2009, Seção 1, p. 20.

Cabrera ML, Kissel DE \& Vigil MF (2005) Nitrogen Mineralization from Organic Residues: Research Opportunities. Journal of Environmental Quality, 34:75-79.

Chalk PM, Magalhães AMT \& Inácio CT (2013) Towards an understanding of the dynamics of compost $\mathrm{N}$ in the soil-plantatmosphere system using ${ }^{15} \mathrm{~N}$ tracer. Plant and Soil, 362:373388 .

CQFS - Comissão de Química e Fertilidade do Solo (2016) Manual de Calagem e Adubação para os Estados do Rio Grande do Sul e de Santa Catarina. Porto Alegre, SBCS. 376p.

Cotta JAO, Carvalho NLC, Brum TS \& Rezende MOO (2015) Compostagem versus vermicompostagem: comparação das técnicas utilizando resíduos vegetais, esterco bovino e serragem. Engenharia Sanitária Ambiental, 20:65-78.

Domínguez J \& Edwards CA (2011) Relationships between composting and vermicomposting. In: Edwards CA, Arancon NQ \& Sherman RL (Eds.) Vermiculture Technology: Earthworms, Organic Waste and Environmental Management. Boca Raton, CRC Press. p.11-25. 
Dores-Silva PR, Landgraf, MD \& Rezende MO de O (2013) Processo de estabilização de resíduos orgânicos: vermicompostagem versus compostagem. Disponível em: <http://quimicanova.sbq.org.br/qn/qnol/2013/vol36n5/04-AR12554.pdf> Acessado em: 30 de julho de 2013.

Ferreira DF (2014) Sisvar: a Guide for its Bootstrap procedures in multiple comparisons. Ciência e Agrotecnologia, 38:109-112.

Frederickson J, Howell G \& Hobson AM (2007) Effect of precomposting and vermicomposting on compost characteristics. European Journal of Soil Biology, 43:S320-S326.

Gómez-Brandón M, Lazcano C, Lores M \& Domínguez J (2010) Detritivorous earthworms modify microbial community structure and accelerate plant residue decomposition. Applied Soil Ecology, 44:237-244.

Gruninger RJ, Puniya AK, Callaghan TM, Edwards JE, Youssef N, Dagar SS, Fliegerova K, Griffith GW, Forster R, Tsang A, McAllister T \& Elshahed MS (2014) Anaerobic fungi (phylum Neocallimastigomycota): advances in understanding their taxonomy, life cycle, ecology, role and biotechnological potential. Federation of European Microbiological Societies. Microbiology Ecology, 90:1-17.

Kiehl EJ (1985) Fertilizantes Orgânicos. Piracicaba, Editora Agronômica "Ceres" Ltda. 492p.

Komilis DP \& Ham RK (2003) The effect of lignin and sugars to the aerobic decomposition of solid wastes. Waste Management, 23:419-423.

Lazcano C, Gómez-Brandón M \& Domínguez J (2008) Comparison of the effectiveness of composting and vermicomposting for the biological stabilization of cattle manure. Chemosphere, 72:1013-1019.

Lim SS, Lee SM, Lee SH \& Choi WJ (2010) Nitrogen isotope compositions of synthetic fertilizer, raw livestock manure slurry, and composted livestock manure. Korean Journal of Soil Science and Fertilizer, 43:453-457.

Lynch DH, Voroney RP \& Warman PR (2006) Use of ${ }^{13} \mathrm{C}$ and ${ }^{15} \mathrm{~N}$ natural abundance techniques to characterize carbon and nitrogen dynamics in composting and in compost-amended soils. Soil Biology \& Biochemistry, 38:103-114.

Malik K, Tokkas J, Anand RC \& Kumari N (2015) Pretreated rice straw as an improved fodder for ruminants-An overview. Journal of Applied and Natural Science, 7:514-520.

Moral R, Paredes C, Bustamante MA, Marhuenda-Egea F\& Bernal MP (2009) Utilisation of manure composts by high-value crops: Safety and environmental challenges. Bioresource Technology, 100:5454-5460.

Murphy J \& Riley JP (1962) A modified single solution method for determination of phosphate in natural waters. Analytica Chimica Acta, 27:31-36

Ngo PT, Rumpel C, Ngo QA, Alexis M, Vargas GV, Gil MLM, Dang DK \& Jouquet P (2013) Biological and chemical reactivity and phosphorus forms of buffalo manure compost, vermicompost and their mixture with biochar. Bioresource Technology, 148:401-407.
Paradelo R, Moldes AB \& Barral MT (2013) Evolution of organic matter during the mesophilic composting of lignocellulosic winery wastes. Journal of Environmental Management, 116:18 -26 .

Rodella AA \& Alcarde JC (1994) Avaliação de materiais orgânicos empregados como fertilizantes. Scientia Agricola, 51:556-562

Santos FG, Escostegy PAV \& Rodrigues LB (2010) Qualidade de esterco de ave poedeira submetido a dois tipos de tratamentos de compostagem. Revista Brasileira de Engenharia Agrícola e Ambiental, 14:1101-1108.

Scherer EE, Nesi CN \& Massotti Z (2010) Atributos químicos do solo influenciados por sucessivas aplicações de dejetos suínos em áreas agrícolas de Santa Catarina. Revista Brasileira de Ciência do Solo, 34:1375-1383.

Silva FC (2009) Manual de análises químicas de solos, plantas e fertilizantes. $2^{a}$ ed. Brasília, Embrapa Informação Tecnológica. $627 \mathrm{p}$.

Sun X, Lu P, Jiang T, Schuchardt F \& Li G (2014) Influence of bulking agents on $\mathrm{CH}_{4}, \mathrm{~N}_{2} \mathrm{O}$, and $\mathrm{NH}_{3}$ emissions during rapid composting of pig manure from the Chinese Ganqinfen system. Journal of Zhejiang University-Science B (Biomedicine \& Biotechnology), 15:353-364.

Swarnam TP, Velmurugan A, Pandey SK \& Roy SD (2016) Enhancing nutrient recovery and compost maturity of coconut husk by vermicomposting technology. Bioresource Technology, 207:76-84.

Tedesco MJ, Gianello C, Bissani CA, Bohnen H \& Volkweiss SJ (1995) Análises de solo, plantas e outros materiais. $2^{a}$ ed. Porto Alegre, Universidade Federal do Rio Grande do Sul. 174p. (Boletim técnico, 5).

Tuomela M, Vikman M, Hatakka A \& Itävaara M (2000) Biodegradation of lignin in a compost environment: a review. Bioresource Technology, 72:169-183.

Van Soest PJ (1963) Use of detergents in the analysis of fibrous feeds. I. Preparation of fiber residues of low nitrogen content. Journal of the Association of Official Agricultural Chemists, 46:825-829.

Woods End (2005) Woods End Research Laboratory Interpreting Waste \& Compost Tests. Disponível em: <http:// www.woodsend.org>. Acessado em: 10 de fevereiro de 2016.

Wu G, Qu P, Sun E, Chang Z, Xu Y \& Huang H (2015) Physical, chemical, and rheological properties of rice husks treated by composting process. BioResources, 10:227-239.

Zeng Y, Guardia A, Daumoin M \& Benoist JC (2012) Characterizing the transformation and transfer of nitrogen during the aerobic treatment of organic wastes and digestates. Waste Management, 32:2239-2247. 